\title{
Caracterización clínica y epidemiológica de Chikungunya en niños
}

\author{
Clinical and epidemiological characterization of Chikungunya in children
}

\author{
José Raúl Valenzuela* Melida Galeas Oliva** Marlin del Carmen Erazo*** Rossana Sánchez****
}

\section{RESUMEN}

Antecedentes y objetivos: La fiebre Chikungunya (CHIKF) es una enfermedad de infección viral causada por un arbovirus (CHIKV), transmitida a los humanos a través de la picadura del mosquito de género Aedes. El objetivo del estudio fué caracterizar clínica y epidemiológicamente los pacientes que presentaron dicha enfermedad. Pacientes y Métodos: Se realizó un estudio descriptivo transversal en el área de emergencia pediátrica del Hospital Regional del Norte - Instituto Hondureño de Seguridad Social (HRN-IHSS). Los datos se obtuvieron de las fichas epidemiológicas de los casos sospechosos de CHIKF que asistieron a la emergencia de pediatría entre los meses de enero y marzo del 2015. Se estudiaron 48 pacientes que cumplieron con los criterios de inclusión. Resultados: El 52\% de los pacientes ingresados provenían del casco urbano de San Pedro Sula (SPS). El $63 \%$ de los casos asistieron al hospital cuando presentaban de 1 a 2 días de evolución. El síntoma que se presentó con mayor frecuencia fue la fiebre (96\%), seguido de rash (71\%), la artritis y artralgias se presentaron en el 58\% de los casos. En el 75\% de los casos había familiares con síntomas similares. Conclusiones: Los lactantes menores y los escolares del sexo masculino fueron los más afectados, y no hubo antecedente de haber realizado viajes previos.

\section{PALABRAS CLAVE}

Aedes, artralgia, Virus Chikungunya.

\section{ABSTRACT}

Background: Chikungunya fever (CHIKF) is a

* Residente de segundo año de Pediatría, Universidad Nacional Autónoma de Honduras-Valle de Sula (UNAH-VS)

**Residente de primer año de Pediatría, UNAH-VS

***Pediatra del Instituto Hondureño de Seguridad Social-Hospital Regional del Norte (IHSS-HRN)

**** Epidemióloga del IHSS-HRN

Dirigir correspondencia a:mjgaleas@gmail.com

Recibido: 11/Jun/2015, aprobado: 22/Jul/2015 disease viral infection caused by an arbovirus (CHIKV) mainly transmitted to humans by mosquitoes of the genus Aedes. A clinical and epidemiological characterization was performed in the patients that presented the disease. Patients and Methods: Across-sectional study was conducted in the department of pediatrics, specifically in the emergency area of the Regional Hospital of North Honduran Social Security Institute (HRN-IHSS). The data was obtained from the epidemiological records of suspected cases of CHIKF who attended the pediatric emergency, between January and March 2015.48 were obtained. Results: $52 \%$ of hospitalized patients lived in the city. In $63 \%$ of cases attended the hospital when they had between 1- 2 days of symptoms.

The main symptoms were fever (96\%), followed by rash (71\%); arthritis and arthralgia occurred in almost $58 \%$ of cases. In $75 \%$ of patients they had family members with similar symptoms. Conclusions: The age at which the symptoms occurred was in young infants and school-predominant in males and no history of previous trips.

\section{KEYWORDS}

Aedes, arthralgia, Chikungunya virus.

\section{INTRODUCCIÓN}

La fiebre de chikungunya (CHIKF) es una enfermedad de infección viral causada por un arbovirus (CHIKV) transmitida principalmente a los humanos a través de la picadura de mosquitos del genero Aedes; principalmente por el Ae. Aegypti y Ae. Albopictus, las mismas especies involucradas en la transmisión del dengue. ${ }^{(1-5)}$

CHIKF se ha catalogado como una enfermedad reemergente en los países del Asia meridional, Asia sudoriental, sur y este de África, siendo la India el país con mayor número de casos reportados. ${ }^{(4-6)}$ 
Actualmente este virus ha provocado brotes en nuevas regiones. Las epidemias de CHIKV han mostrado históricamente una presentación cíclica, con períodos inter epidémicos que oscilan entre 4 y 30 años. ${ }^{(4,7-10)}$

En los años 1770 se reportaron epidemias de fiebre, rash y artritis semejantes a CHIK, sin embargo, el virus no se aisló de suero humano y de mosquitos hasta que ocurrió una epidemia en Tanzania en 1952-1953.

El nombre chikungunya deriva de una palabra en Makonde, el idioma que habla el grupo étnico Makonde que vive en el sudeste de Tanzania y el norte de Mozambique, significa a grandes rasgos "aquel que se encorva" ha causado brotes en el este de África (Tanzania y Uganda), en África Austral (Zimbabwe), África Oriental, África occidental (Senegal), África central (República Centroafricana y República Democrática del Congo).

En el 2004 el virus produjo brotes en muchos territorios nuevos de las islas del Océano Indico y en Italia. ${ }^{(6,11-15)}$ A partir de esa fecha se han reportado brotes intensos y extensos en África, Islas del Océano Índico, la región del Pacífico, incluyendo Australia y Asia.

En el 2007 el virus se extendió a Italia y las Américas, así mismo se registraron casos importados en Estados Unidos, Canadá, Guyana Francesa, Martinica, Guadalupe y Brasil.

Entre el 3 y $28 \%$ de los casos la infección puede ser asintomática, lo que contribuye a su diseminación. ${ }^{(8,16-22)}$

Afecta a todos los grupos de edad y ambos sexos. El período de incubación es de 3 a 7 días. (Rango: 1-12 días). Se caracteriza por fiebre de inicio brusco, artritis, artralgias y erupción. ${ }^{(15,19,}$ 23-24)

El mecanismo principal de transmisión consiste en la picadura de mosquitos Aedes aegypti o Aedes albopictus y en menor frecuencia por transmisión vertical (madre sintomática 4 días antes y 2 días después del parto) pinchazo con agujas y transfusiones sanguíneas. ${ }^{(17,25-29)}$

El objetivo del estudio fue caracterizar clínica y epidemiológicamente a los niños con chicungunya en el HRN IHSS, durante el período de enero a marzo 2015.

\section{PACIENTES Y MÉTODOS}

El estudio se realizó en el HRN-IHSS. Estudio descriptivo retrospectivo transversal realizado en el área de emergencia pediátrica. Durante el periodo de enero a marzo de 2015, hablamos de caso sospechoso en todo paciente con fiebre $\geq$ $38.5^{\circ} \mathrm{C}$ y artralgia severa o artritis, de comienzo agudo, que no se explican por otras condiciones médicas, que presenta rash, y que reside o ha visitado áreas endémicas o epidémicas durante las dos semanas anteriores al inicio de síntomas; y de caso confirmado cuando se tiene un caso sospechoso con resultado positivo por RT-PCR o ELISA, ${ }^{(2,23-25)}$ se ingresaron 167 casos de síndromes febriles, de los cuales 48 cumplían con los criterios como sospechosos para fiebre por Chikungunya y a quienes se les llenó la ficha epidemiológica establecida y proporcionada por la Secretaria de Salud. Se incluyeron todos los niños y niñas desde 1 día de vida hasta los 18 años de edad, que presentaban características clínicas y epidemiológicas de Chikungunya, y que fueron ingresados en dicha institución, se excluyeron a los pacientes que presentaban una patología infecciosa ya definida y que no reunían los criterios epidemiológicos para Chikungunya.

La información obtenida se procesó en el software estadístico Epi Info 3.02 con el posterior análisis estadístico, se aplicaron medidas de tendencia central, para la presentación de los datos se utilizó la hoja de Microsoft Excel 2010.

\section{RESULTADOS}

Se obtuvieron 48 fichas epidemiológicas en niños con sospecha de Chikungunya. El 69\% se trató de pacientes del género masculino y el $31 \%$ del femenino; el rango de edad predomi- 
nante de los niños afectados con Chikungunya fue entre los 28 días y un año de nacido, seguidos de los de edad escolar (6 -12 años).

Ver tabla 1

Tabla No. 1: Distribución de los pacientes según la edad

\begin{tabular}{lcc}
\hline Edad & $\mathbf{n = 4 8}$ & Porcentaje \\
\hline$<28$ días & 4 & $8 \%$ \\
28 días -1 año & 17 & $35 \%$ \\
1 año-2 años & 2 & $4 \%$ \\
2años-6años & 9 & $19 \%$ \\
6años-12años & 16 & $33 \%$ \\
$>$ 12 años & 0 & $0 \%$ \\
\hline
\end{tabular}

En cuanto a la procedencia de los pacientes, se encontró que el 52\% proceden del casco urbano. (Tabla No. 2)

Tabla No. 2. Procedencia de los pacientes como sospechosos de Fiebre por Chikungunya

\begin{tabular}{lcc}
\hline \multicolumn{1}{c}{ Municipio } & $\mathbf{n = 4 8}$ & Porcentaje \\
\hline SPS & 25 & $52 \%$ \\
Choloma & 12 & $25 \%$ \\
Villanueva & 8 & $16.6 \%$ \\
Lima & 2 & $4.1 \%$ \\
San Manuel & 1 & $2 \%$ \\
\hline
\end{tabular}

El $63 \%$ de los pacientes que acudieron en busca de asistencia médica, lo hizo durante los primeros 2 días del cuadro sintomático.

Ver tabla 3

Tabla No. 3. Número de días con síntomas, cuando acudieron al centro asistencial

\begin{tabular}{ccc}
\hline $\begin{array}{c}\text { Número de } \\
\text { días }\end{array}$ & $\mathbf{n = 4 8}$ & Porcentaje \\
\hline 1-2 días & 30 & $63 \%$ \\
3-5 días & 15 & $31 \%$ \\
$>$ 5 días & 3 & $6 \%$ \\
\hline
\end{tabular}

El $75 \%$ de los pacientes hacían alusión que conocían personas en su comunidad o familia que presentaban síntomas similares.

Se interrogó acerca de viajes realizados a áreas endémicas en los 14 días previos a los síntomas, encontrando que un $87 \%$ no realizó ningún desplazamiento fuera de la ciudad y el $13 \%$ restante se movilizó hacia las ciudades de Choluteca, Santa Bárbara y Yoro.

El síntoma que se presentó con mayor frecuencia fue la fiebre (96\%), seguido de rash (71\%), la artritis y artralgias se presentaron en el $58 \%$ de los casos. (Tabla 4)

Tabla No.4. Síntomas más frecuentes

\begin{tabular}{lcc}
\hline \multicolumn{1}{c}{ Signos y Síntomas } & $\mathbf{n = 4 8}$ & \% \\
\hline Fiebre & 46 & 96 \\
Rash- Máculo Papular & 34 & 71 \\
Artralgias & 28 & 58 \\
Mialgias & 23 & 48 \\
Artritis & 17 & 35 \\
Vomitos & 16 & 33 \\
Cefalea & 16 & 33 \\
Nauseas & 13 & 27 \\
Astenia & 10 & 21 \\
Rash Vesicular & 7 & 14 \\
Dolor de Espalda & 7 & 14 \\
Otras & 4 & 8.3 \\
Edema & 3 & 6.2 \\
Meningoencefalitis & 2 & 4.2 \\
Conjuntivitis & 1 & 2.1 \\
\hline
\end{tabular}

\section{DISCUSIÓN}

La fiebre por Chikungunya es una infección reciente en las Américas, los primeros casos reportados fueron en diciembre del 2013 en contraste con los continentes de Asia y África los cuales presentan casos de dicha enfermedad desde 1952 donde fue identificado y aislado el virus en Tanzania, los recientes casos publicados en América Central nos hacen 
pensar de que nuestro país no está exento de padecer dicha enfermedad como se ha demostrado en los últimos meses, observando una rápida propagación de la misma. Esto se puede considerar como una particularidad ya que el vector transmisor se reproduce en sitios con clima tropical, subtropical y templado la cual se comparte con dichos continentes. ${ }^{(21-22,30-31)}$

La enfermedad tal como se ha mencionado en la literatura mundial, se da en sitios de hacinamiento y de poca salubridad, lo que concuerda con nuestro estudio ya que San Pedro Sula se encuentra en un área endémica, siendo el brote de mayor porcentaje de casos en el casco urbano con un $57 \%$ en contraste con el resto de zonas aledañas urbano-rurales. ${ }^{(22,32-34)}$

Se evidenció que los pacientes mayormente afectados fueron los lactantes y los preescolares (de 28 días a 1 año y de 2 a 6 años respectivamente), representando estos el $68 \%$ de los casos. Esto se debe a la vulnerabilidad de los lactantes y de los niños en edad escolar, teniendo como factor de riesgo el hacinamiento y la insalubridad de los centros educativos, aunado del no acato de los padres a las recomendaciones generales para la prevención de esta enfermedad; el género predominante fue el masculino con un $69 \%$ a diferencia de lo expuesto en la bibliografía consultada donde no se encontró predominancia por género. ${ }^{(35-37)}$

Se evidencio mayor susceptibilidad a desarrollar complicaciones entre los pacientes de menor edad, siendo los neonatos el grupo de mayor riesgo; encontrándose dos casos de Meningoencefalitis; la cual es una de las manifestaciones atípicas graves secundarias a la fiebre por Chikungunya, cabe resaltar que la incidencia de manifestaciones atípicas según la literatura mundial es del $0.3 \% .^{(1-2,38)}$

El principal síntoma que se manifestó fue la fiebre con un $96 \%$, valor similar al reportado por la OMS y OPS en las Américas con un porcentaje entre el 76 y el 100\%.(3-4,39) Pero además se observó que el segundo síntoma o signo mas frecuente fue el rash maculopapular en un $71 \%$, a diferencia de lo descrito en la bibliografia consultada que menciona las artralgias en primer lugar, seguido por el rash en un rango del 22 - al $70 \%$, esto quizás por la difícil percepción de este síntoma en niños menores de 1 año. ${ }^{(1-2,19,40)}$

En conclusión, múltiples factores se han asociado con el surgimiento de la fiebre por Chikungunya, entre ellos la evolución del virus, las condiciones socioeconómicas, el cambio en las características del vector y el aumento del comercio mundial y los viajes.

Con un aumento en la incidencia y el número de casos atípicos graves, la pregunta ya no es si se produce otra epidemia, sino más bien cuándo y cómo estar preparado para ello.

Existe una necesidad de desarrollar una mejor estrategia de prevención y manejo, y de esa forma disminuir el excesivo gasto que implica el surgimiento de esta enfermedad. ${ }^{(1-4,39-40)}$

\section{BIBLIOGRAFÍA}

1. Guía de preparación y respuesta ante la eventual introducción del virus Chikungunya en las América. [internet] Washinton, DC: Organización Panamericana de la Salud; 2011. [citado 2015 May 22] Disponible en: http://www1.paho.org/hq/dmdo cuments/CHIKV_Spanish.pdf.
2. Guía de Manejo Clínico de Fiebre Chikungunya. [internet] Tegucigalpa, MDC: Secretaría de Salud de Honduras; 2012. [citado 2015 May 22] Disponible en:

http://www.salud.gob.hn/noticias/bole tin\%20mayo2014/guiamanejoclinicochik v.pdf. 
3. Guideline for Clinical Management of Chikungunya Fever. [internet] India: World Health Organization; 2008. [citado 2015 May 22] Disponible en: http:// www.wpro.who.int/mvp/topics/ntd/Clini cal_Mgnt_Chikungunya_WHO_SEARO.pdf.

4. Staples J, Breiman R, Powers A. Chikungunya fever: an epidemiological review of a re-emerging infectious disease. Clin Infect Dis. 2009; 49(6):942-948. DOI: 10.1086/ 605496.

5. Economopoulou A, Dominguez M, Helynck B, Sissoko D, Wichmann O, Quenel P, et al. Atypical chikungunya virus infections: clinical manifestations, mortality and risk factors for severe disease during the 2005-2006 outbreak on Reunion. Epidemiol Infect. 2009; 137(4):534-541. DOI:10.1017/ S0950268808001167.

6. Frank C, Schoneberg I, Stark K. Trends in Imported Chikungunya Virus Infections in Germany. Vector Borne Zoonotic Dis. 2011 Jun; 11(6):631-636. DOI: 10.1089/vbz.2010. 0269.

7. Josseran L, Paquet C, Zehgnoun A, Caillere N, Le Tertre A, Solet J, et al. Chikungunya disease outbreak, Reunion Island. Emerg Infect Dis. 2006 Dec; 12(12):1994-1995. DOI: 10.3201/eid1212.060710.

8. Lahariya C, Padhan SK. Emergence of chikungunya virus in Indian subcontinent after 32 years: a review. J Vect Borne Dis [internet]. 2006 Dec [citado 2015 May 22]; 43:151-160. Disponible en: http:// www.mrcindia.org/journal/issues/434151. PDF.

9. Borgherini G, Poubeau P, Staikowsky F, Lory M, Le Moullec N, Becquart J, et al. Outbreak of chikungunya on Reunion Island: early clinical and laboratory features in 157 adult patients.. Clin Infect Dis. 2007 Jun 1; 44(11):1401-1407. DOI: 10.1086/517537.
10. Queyriaux B, Simon F, Grandadam M, Michel $\mathrm{R}$, Tolou $\mathrm{H}$, Boutin J. Clinical burden of chikungunya virus infection. Lancet Infect Dis. 2008 Jan; 8(1):2-3. DOI: http://dx.doi. org/10.1016/S1473-3099(07)70294-3.

11. Seetharam K, Sridevi K, Vidyasagar P. Cutaneous Manifestation of Chikungunya Fever. Indian Pediatrics.[internet] 2012 [citado 2015 May 23]; 49:51-53. Disponible en: http://www.indianpediatrics.net/jan2012/ jan-51-53.htm.

12. Martín-Farfán A, Calbo-Torrecillas F, Pérezde PI. Fiebre importada por el virus de Chikungunya. Enferm Infecc Microbiol Clin. 2008; 26(6):343-344. DOI: 10.1157/ 13123 839.

13. Sebastian M, Lodha R, Kabra S. Chikungunya infection in children. Indian J Pediatr. 2009 Feb; 76(2):185-189. DOI: 10.1007/ s12098-009-0049-6.

14. Pialoux G, Gaüzère B, Jauréguiberry $S$, Strobel M. Chikungunya, an epidemic arbovirosis. Lancet Infect Dis. [Internet] 2007 May[citado 11 Jun 2014]; 7(5):319- 327. Disponible en: http://dx.doi.org/10.1016/S1473-30 99(07)70107-X.

15. Appassakij H, Khuntikij P, Kemapunmanus M, Wutthanarungsan R, Silpapojaku IK.Viremic profiles in asymptomatic and symptomatic chikungunya fever: a blood transfusion threat? Transfusion. 2013 Octuber; 53 (10Pt2): 2567-74. DOI: 10.1111/j.1537-2995. 2012.03960.x.

16. Laoprasopwattana K, Kaewjungwad L, Jarumanokul R, Geater A. Differential Diagnosis of Chikungunya, Dengue Viral Infection and Other Acute Febrille Illnesses in Children. Pediatr Infect Dis J. 2012 May; 31(5): 459-463. DOI: 10.1097/INF.0b013e31824 bb06d.

17. Krishnamoorthy $K$, Harichandrakumar KT, Krishna Kumari A, Das LK. Burden of chikun- 
gunya in India: estimates of disability adjusted life years (DALY) lost in 2006 epidemic. Journal of Vector Borne Diseases [internet] 2009; 46: 26-35. [citado 2015 May 22] Disponible en: http://www.mrcindia.org/ journal/issues/461026.pdf.

18. Ruiz D, Sánchez I, Olson K, Harrington L. Modeling Dynamic introduction of Chikungunya Virus in the United States. Plos Negl Trop Dis. 2012; 6(11):1918-1924. DOI: 10. 1371/journal.pntd.0001918.

19. Gérardin P, Fianu A, Michault A, Mussard C, Bousaï k, Rollot $\mathrm{O}$, et al. Predictors of Chikungunya rheumatism: a prognostic survey ancillary to the TELECHIK cohort study. Arthritis Research \& Therapy 2013, 15:R9 DOI: 10.1186/ar4137.

20. Robinson MC. Una epidemia de virus de la enfermedad en el sur de provincia, territorio de Tanganyika, en 1952-53. Trans $\mathrm{R}$ Soc Trop Med Hyg 1955; 49 (1): 28-32.

Disponible en: http://www.ncbi.nlm.nih. gov/pub med/14373835.

21. Moro ML, Gagliotti C, Silvi G, et al. Chikungunya virus in North- Eastern Italy: a seroprevalence survey. Am J Trop Med Hyg. Mar 2010; 82(3): 508-511. doi: 10.4269/ajtmh. 2010.09-0322.

22. Paquet C, I Quatresous, JL Solet, D Sissoko, Renault P, Pierre V, H Cordel, Lasalle C, Thiria J, Zeller H, SchuF- fnecker I. brote de Chikungunya en la Reunión: epidemiológica logía y vigilancia Euro Surveill 2006; 11:2.

Disponible en: http://www.eurosurveillan ce.org/ViewArticle.aspx?Articleld=2891.

23. Manimunda SP, Vijayachari P, Uppoor R, Sugunan AP, Singh SS, Rai SK et. al.Clinical progression of chikungunya fever during acute and chronic arthritic stages and the changes in joint morphology as revealed by imaging. Trans R Soc Trop Med Hyg 2010; 104:392-399. doi: 10.1016/j.trstmh. 2010.01.011.
24. Kularatne S, Gihan M, Weerasinghe S, Gunasena S. Concurrent outbreaks of Chikungunya and Dengue fever in Kandy, Sri Lanka, 2006-07: a comparative analysis of clinical and laboratory features. Pstgrad Med J 2009; 85:342-46. doi: 10.1136/pgmj. 2007.066746.

25. European Centre for Disease Prevention and Control. Mission Report Chikungunya in Italy, Joint ECDC/WHO visit for a European risk assessment 17-21 September 2007. Italy: European Centre for Disease Prevention and Control; 2007. Disponible en: http://ecdc.europa.eu/en/healthtopics/ Documents/0709_Chikungunya_fever_ Mission_report.pdf.

26. Chhabra M, Mittal V, Bhattacharya D, Rana UVS, Lal S. Chikungunya fever: A re-emerging viral infection. National Institute of Communicable Diseases, New Delhi, India [Internet]. 2008 [Consulta el 25 de marzo 2013]; 26(2)5-12. Disponible en: http:// www.ijmm.org/article.asp?issn $=0255$ 0857; year $=2008$; volume $=26$; issue $=1$; spage $=5$; epage $=12$; aulast $=$ Chhabra .

27. Mahendradas $\mathrm{P}$, Avadhani K, Shetty R. Chikingunya and the eye: a review. Journal of Ophthalmic Inflammation and Infection [Internet]. 2013 [Consulta el 27 de marzo 2013]; 3:35. Disponible en: http://www. joii-journal.com/content/3/1/35.

28. Parida MM, Santhosh SR, Dash PK, Lakshmana Rao PV. Rapid and Real-time Assays for Detection and Quantification of Chikungunya Virus. Future Virology [Internet]. 2008 [Consulta el 27 de marzo 2013]; 3(2) 179-92. Disponible en: http://www.future medicine.com/doi/abs/10.2217/17460794. 3.2.179? journalCode $=f v l$.

29. Chia P, MAh-Lee M, Hann Chu J.Chikungunya fever: A review of a re-emerging mosquito-borne infectious disease and the current status. En Current Research, Technology 
and Education Topics in Applied Microbiology and Microbial Biotechnology. Singapure: A Mendez-Vilas (Ed); 2010. P. 597-606. Disponible en: http://www.formatex.info/ microbiology2/597-606.pdf.

30. Naze F, Le Roux K, Schuffenecker I, Zeller H, Staikowsky F, Grivard P et. al. Simultaneous detection and quantitation of Chikungunya, Dengue and West Nile viruses by multiplex RT-PCR assays and Dengue virus typing using High Resolution Melting. J Viro Met Elsevier 2009; 162: 1-7. Disponible en: http://www.sciencedirect.com/science/ article/pii/S0166093409001074.

31. Gaüzère B. Infection à virus Chikungunya. Médicine Tropicale, Diplôme de Médicine Tropicale des Pays de l'Océan Indien (La Réunion) 2011.p 1-7.

32. Chopra A, Anuradha V, Ghorpade R. Saluja M. Acute Chikungunya and persistent muscoloskeletal pain following the 2006 Indian epidemic: a 2-year prospective rural community study. Epidemiol. Infect 2012; 140:842-50. Disponible en: http://www.nc bi.nlm.nih.gov/pmc/articles/PMC4181148/.

33. Lewthwaite $P$, Vasanthapuram $R$, et al. Chikungunya Virus and Central Nervous System Infections in Children, India. Emerg Infect Dis. 2009 February; 15(2): 329-331. Disponible en: http://wwwnc.cdc.gov/eid/ article/15/2/08-0902_article.

34. Jansen A, Franck C, et al. Surveillance of vector-borne diseases in Germany trends and challenges in the view of disease emergence and climate change. Parasite Res (2008) (Suppl 1) 103: S11-S17. Disponible en: http://link.springer.com/article/10.1007 \%2Fs00436-008-1049-6.

35. Bouquillard É, Combe B. A report of 21 cases of reumatoid arthritis following Chikungunya fever. A mean follow u-p of two years. Joint Bone Spine 2009; 76:654-57. doi: 10.1016/j.jbspin.2009.08.005.

36. Thiboutot M, Kannan S, Kawalekar O, Shedlock D, Khan A, Sarangan G, et. al. Chikungunya: A Potentially Emerging Epidemic?. PLoS Negl Trop Dis [Internet]. 2010 [Consulta el 1 abril 2013]; 4(4):e623. DOI: 10.1371/ journal.pntd.0000623.

37. Kamath S, Das AK, Parikh F. Chikungunya. Japi 2006; 54:725-26. Disponible en: http:// europepmc.org/abstract/med/172 12022.

38. Mahendradas P, Shetty R, Malathi J, Madhavan $H$. Chikungunya virus iridocyclitis in Fuchs' heterochromic iridocyclitis. Indian J Ophthalmo [Internet]. 2010 [Consulta el 1 Abril 2013]; 58(6):545-47. Disponible en: http://www.ncbi.nlm.nih.gov/pmc/arti cles/PMC2993993/.

39. Lenhart A, Orelus N, Maskill R, Alexander N, Streit T, McCall PJ. Insecticide-treated bednets to control dengue vectors: preliminary evidence from a controlled trial in Haiti. Trop Med Int Health. 2008; 13(1):56-67. DOI: 10.1111/j.1365-3156.2007.01966.x.

40. Marano C, Freedman D. Global health surveillance and travelers' health. Current Opinion in Infectious Diseases 2009; 22: 423-29. doi: 10.1097/QCO.0b013e32832 ee89. 